\title{
A comparison of home care quality indicator rates in two Canadian provinces
}

\author{
Amanda M Mofina and Dawn M Guthrie
}

\begin{abstract}
Background: Home care is becoming an increasingly vital sector in the health care system yet very little is known about the characteristics of home care clients and the quality of care provided in Canada. We describe these clients and evaluate home care quality indicator rates in two regions.

Methods: A cross-sectional analysis of assessments completed for older (age 65+) home care clients in both Ontario ( $n=102,504)$ and the Winnipeg Regional Health Authority $(n=9,250)$ of Manitoba, using the Resident Assessment Instrument for Home Care (RAl-HC). This assessment has been mandated for use in these two regions and the indicators are generated directly from items within the assessment. The indicators are expressed as rates of negative outcomes (e.g., falls, dehydration). Client-level risk adjustment of the indicator rates was used to enable fair comparisons between the regions.
\end{abstract}

Results: Clients had a mean age of 83.2 years, the majority were female (68.6\%) and the regions were very similar on these demographic characteristics. Nearly all clients (92.4\%) required full assistance with instrumental activities of daily living (IADLs), approximately 35\% had activities of daily living (ADL) impairments, and nearly 50\% had some degree of cognitive impairment, which was higher among clients in Ontario (48.8\% vs. $37.0 \%)$. The highest quality indicator rates were related to clients who had $\mathrm{ADL} /$ rehabilitation potential but were not receiving therapy (range: 66.8\%-91.6\%) and the rate of cognitive decline (65.4\%-76.3\%). Ontario clients had higher unadjusted rates across 18 of the 22 indicators and the unadjusted differences between the two provinces ranged from $0.6 \%$ to $28.4 \%$. For 13 of the 19 indicators that have risk adjustment, after applying the risk adjustment methodology, the difference between the adjusted rates in the two regions was reduced.

Conclusions: Home care clients in these two regions are experiencing a significant level of functional and cognitive impairment, health instability and daily pain. The quality indicators provide some important insight into variations between the two regions and can serve as an important decision-support tool for flagging potential quality issues and isolating areas for improvement.

Keywords: Quality indicators, Home care, Standardized assessment, RAI-HC

\section{Background}

A $50 \%$ increase in the number of individuals receiving home care has been observed in Canada within the last decade largely due to the essential role home care plays in primary care and chronic disease management [1]. Home care in Canada costs $\$ 3.4$ billion annually, [2] and approximately $80 \%$ of home care clients are 65 years of age or older [3]. In the US, home care costs in 2010 were in excess of $\$ 70$ billion [4], and similar to Canada,

\footnotetext{
* Correspondence: dguthrie@wlu.ca

Department of Kinesiology and Physical Education, Wilfrid Laurier University, 75 University Ave. W, Waterloo, ON N2L 3C5, Canada
}

the majority of clients were older adults [5]. Based on this trend, home care could expect an increase in the volume of clients in the upcoming years with the escalation in the number of older adults with chronic conditions. Home care services will have to function both efficiently and effectively to provide optimal services to the greatest number of clients. In order to achieve this goal, quality of care should be continually assessed in order to support ongoing continuous quality improvement. However, in Canada, little is currently known about home care clients or the quality of care they receive [6-10].

\section{Ciomed Central}

(c) 2014 Mofina and Guthrie; licensee BioMed Central Ltd. This is an open access article distributed under the terms of the Creative Commons Attribution License (http://creativecommons.org/licenses/by/2.0), which permits unrestricted use, distribution, and reproduction in any medium, provided the original work is properly cited. 
Home care in Canada is publicly funded and is financed through the provincial government [1]. The provincial/ territorial ministries provide funding, ensure compliance with policies and administer legislation and regulations. Home care in Canada is not standardized across all provinces and, unlike most other health care services, it is not regulated by the Canada Health Act (1984) [11].

In several US states (e.g., Michigan, New Jersey, Massachusetts, North Carolina) and in multiple regions in Canada, including Ontario and in the Winnipeg area of Manitoba (Winnipeg Regional Health Authority or WRHA), the Resident Assessment Instrument for Home Care (RAI-HC) is being used for assessing home care clients in a standardized fashion. The RAI-HC was developed by interRAI (www.interrai.org), an international group of researchers and clinicians who continually develop and refine standardized assessment instruments for older adults and individuals with disabilities [12]. The RAI-HC is an assessment system that includes a set of Clinical Assessment Protocols [13]. Some items within the assessment act as a link to the CAPs by identifying clients at risk of various negative outcomes such as cognitive decline, pain and falls. The CAPs are generated with computer software and there is also written documentation for each CAP that provides the assessor with guidance in terms of further assessment and development of a care plan (see Table 1 for detailed descriptions of the CAPs).

Although the RAI-HC was developed primarily to guide care planning, items within the assessment can also be used to generate a set of 22 home care quality indicators (HCQIs) [7]. These indicators are generated using computer software and represent potential quality issues, but should not be thought of as definitive measures of quality. The indicators were developed to act as a flag, such that high rates on the indicators would necessitate the need for further exploration by home care staff to better understand what factors were influencing the rate (e.g., processes of care). Among the 22 HCQIs, 16 are prevalence-based and the remaining six are incidencebased indicators of quality. Prevalence-based indicators allow for examination of quality at a single point in time. Incidence-based measures, however, reflect client changes in status since they incorporate data at two points in time and represent new instances of the quality issue [7]. The set of $22 \mathrm{HCQIs}$ can highlight potential quality issues and can also be used to monitor changes over time resulting from quality improvement interventions implemented by home care organizations.

For 19 of the indicators, client-level risk adjusters have been proposed to allow for fair comparisons between providers [7]. Clients experiencing poorer health or co-morbidity typically develop more complications and, as a result, have worse outcomes. Risk adjustment is a statistical technique used to attempt to control for the differences in the level of risk between clients residing in different regions. Case-mix differences across regions can lead to inaccurate perceptions of quality and risk adjustment is a method used to attempt to "level the playing field" across different regions [14]. For example, for the prevalence of weight loss indicator, two risk adjusters have been suggested, namely, more severe ADL impairment and a diagnosis of cancer. To date, very few studies have been completed to examine the influence of risk adjustment [8] or to explore how these indicators vary with characteristics of the provider organizations [15].

There is the potential for the HCQIs to be used for a variety of purposes and to reach multiple target audiences. For example, the HCQIs can be used for reports to guide internal quality improvement [7]. Currently in Ontario, several of the HCQIs are being used by Health Quality Ontario, which is funded by the provincial government, to provide public information on home care providers across the province [16]. Although this is a fairly new initiative, these reports have the potential to benefit both the client and home care organization by highlighting providers where quality issues may be of concern and also by identifying top performers whose policies and practices could serve as important models for other providers.

To date, the RAI-HC has been mandated for use in multiple regions across Canada (e.g., Ontario, British Columbia, Yukon Territory) yet little Canadian evidence exists that describes home care clients and rates of quality issues by utilizing standardized data available from this assessment $[7,17]$. The current study was designed to address this gap by providing a comprehensive description of home care clients in these two regions and also examines the HCQI rates between these regions and how the risk adjustment process influenced these rates.

\section{Methods}

For the current study, data from Ontario and the WRHA were chosen based on several criteria including the fact that they are two of the four provinces who have submitted RAI-HC data to a national data warehouse (held by the Canadian Institute for Health Information), they have submitted a large number of assessments and they have the longest history of RAI-HC implementation in Canada. Trained health professionals (typically nurses) collected the data as part of normal practice using the RAI-HC on laptop computers. The RAI-HC was administered on admission and re-assessments were completed every 6 months or upon significant clinical change. The completed assessments were uploaded into their local database and the two regions then submitted the data to the Canadian Institute for Health Information. This project represented secondary analysis of anonymized RAI-HC data from 2006-2010. As such, institutional 
Table 1 Description of clinical assessment protocols [13]

\begin{tabular}{|c|c|}
\hline CAP & Description \\
\hline $\begin{array}{l}\text { Activities of daily living/rehabilitation } \\
\text { potential }\end{array}$ & Identify clients with the potential for improved independence in activities of daily living or self-care \\
\hline Instrumental activities of daily living (IADLs) & $\begin{array}{l}\text { Identify clients who could improve on IADLs (e.g., meal preparation, managing finances or } \\
\text { medications) }\end{array}$ \\
\hline Health promotion & To promote healthy behaviours such as increased physical activity and smoking cessation \\
\hline Institutional risk & $\begin{array}{l}\text { Identify individuals with a high risk of institutionalization and suggests ways to help them stay in the } \\
\text { community }\end{array}$ \\
\hline Communication disorders & $\begin{array}{l}\text { Recognize communication issues and provide strategies to aide in effective communication between } \\
\text { clients and others }\end{array}$ \\
\hline Visual function & Evaluate clients with new or existing vision impairments \\
\hline Alcohol use and hazardous drinking & $\begin{array}{l}\text { Identify alcohol use that may be excessive or at a level that might put client at risk of negative } \\
\text { outcomes }\end{array}$ \\
\hline Cognition & Determine whether cognitive problems are present, either acute or long-standing \\
\hline Behaviour & Identify persons with behavioral symptoms such as wandering, being verbally or physically abusive \\
\hline Depression and anxiety & $\begin{array}{l}\text { Assist in identifying persons who exhibit signs/symptoms of anxiety or depression and put forward } \\
\text { potential treatment options }\end{array}$ \\
\hline Elder abuse & $\begin{array}{l}\text { Identify clients who are living in abusive or neglectful conditions or who are risk of being in this } \\
\text { situation }\end{array}$ \\
\hline Social function & Assist with maintaining or restoring meaningful social connections \\
\hline Cardio-respiratory & $\begin{array}{l}\text { Identify problems of the cardiovascular or respiratory systems that require intervention by a medical } \\
\text { professional }\end{array}$ \\
\hline Dehydration & Alert the professional to clients experiencing dehydration or clients who are at risk of this outcome \\
\hline Falls & Identify recent fall occurrences and if the client is at risk of falling in the future \\
\hline Nutrition & Detection of malnutrition and clients who may be at risk for poor nutrition \\
\hline Oral health & $\begin{array}{l}\text { Detect oral health problems that may be leading to outcomes such as pain, difficulty with eating/ } \\
\text { talking, poor nutrition or a lack of enjoyment of food }\end{array}$ \\
\hline Pain & Identify clients with pain that may result in impairments in everyday function \\
\hline Pressure ulcers & $\begin{array}{l}\text { Assist in identifying clients who have pressure ulcers or skin breakdown or who are at risk of this } \\
\text { outcome }\end{array}$ \\
\hline Skin and foot conditions & $\begin{array}{l}\text { Identify persons who have skin conditions or issues related to the health of their feet (e.g., corns, } \\
\text { bunions, fungi) or who are at risk of developing them }\end{array}$ \\
\hline Adherence & Identify whether issues of non-adherence to suggested treatments/therapies is present \\
\hline Brittle supports & Identify families who may be having difficulty continuing in their caregiving role \\
\hline Medication management & $\begin{array}{l}\text { Compile a list of all medications (both prescription and over-the-counter) being taken and asses if the } \\
\text { client is at risk of inappropriate drug therapy }\end{array}$ \\
\hline Palliative care & Evaluate whether a palliative approach to care is warranted \\
\hline $\begin{array}{l}\text { Preventative health care measures: } \\
\text { immunization and screening }\end{array}$ & $\begin{array}{l}\text { Identify clients who have not received preventative strategies such as blood pressure check, } \\
\text { vaccinations }\end{array}$ \\
\hline Psychotropic drugs & $\begin{array}{l}\text { Identify individuals taking psychotropic drugs and who might benefit a review of their medications by } \\
\text { a physician }\end{array}$ \\
\hline Reduction of formal services & $\begin{array}{l}\text { Evaluate the formal services being delivered currently and determine if the current formal services are } \\
\text { beneficial or could be modified }\end{array}$ \\
\hline Environmental assessment & Identify conditions in the home that may be hazardous and compromise client safety \\
\hline Bowel management & Evaluate problems in bowel functioning as well as gastrointestinal issues \\
\hline $\begin{array}{l}\text { Urinary incontinence and indwelling } \\
\text { catheter }\end{array}$ & $\begin{array}{l}\text { To identify when incontinence is present or a catheter is being used and address the underlying } \\
\text { causes of incontinence }\end{array}$ \\
\hline
\end{tabular}

ethics review was not required. These data were accessed free of charge through the Graduate Student Data Access Program (the first author was a graduate student at the time this project began). The application to request access to these data involved a description of the current project and required data, intentions regarding dissemination of results as well as computer security confirmation. 
From the original dataset $(n=785,169)$ the sample was restricted to clients who were at least 65 years of age, had at least two assessments (for the incidence-based HCQIs) or those clients with one assessment that was not their initial assessment (for prevalence-based HCQIs). Only re-assessments were used in calculating the prevalence indicators since the home care providers would not have had time to implement a care plan for these clients and this could negatively affect the HCQI rates [7].

Additionally, the Ontario sample was further restricted to assessments completed in 2010 ( $n=102,554)$, the most recent year of data available from the Canadian Institute for Health Information. The final dataset $(n=174,112$ assessments) included 9,250 unique assessments from clients from Manitoba, specifically, from the WRHA since this was the only health region submitting data to the Canadian Institute for Health Information. To maximize the sample size in the WRHA, the most recent data available from the Canadian Institute for Health Information were used (2006-2007). We did not have access to Ontario data for 2006-2007 to align with the Winnipeg sample, and therefore chose instead to use the most recent data available for Ontario (2010). Where multiple assessments were available (9.1\% of assessments in Winnipeg and $14.6 \%$ in Ontario), the most recent assessment was kept for analysis, to better reflect each client's current health status.

Embedded within the RAI-HC are a number of health sub-scales that are generated directly from items within the assessment that have been previously validated. For example, the Depression Rating Scale summarizes seven items and has been shown to be a valid indicator of clinically relevant signs/symptoms of depression [18,19]. The Cognitive Performance Scale has been validated against the Mini-Mental State Examination [20,21]. The Activities of Daily Living Self-performance Hierarchy Scale is a valid and reliable measure of the client's level of independence across four ADL items. It ranges from zero to six, with a higher score indicating a greater level of dependence on others [22]. The Changes in Health End-stage Disease Signs and Symptoms scale measures health instability and higher scores have been shown to be related to an increased risk of mortality [23,24]. The Pain Scale is based on two pain items related to the frequency and severity of daily pain. It can range from 0 (no pain) to 3 (severe daily pain) and has shown to be highly predictive of pain on the Visual Analogue Scale in nursing home residents [25].

T-tests or chi-square analyses were used, as appropriate, to compare the provinces on demographic characteristics (e.g., sex), the health sub-scales and the CAPs. An absolute difference between regions of at least 10\% was used to define differences that were clinically relevant. Given the large sample size, very small and likely meaningless differences would be statistically significant with an alpha level of 0.05 .

The HCQIs were all calculated based on items within the RAI-HC and are typically expressed as rates of issues to be avoided in the home care population [7]. Each HCQI is defined by both a numerator and a denominator, with the denominator used to identify the population at risk. For example, the inadequate pain control HCQI is calculated by dividing the number of clients who experience pain that is not adequately controlled by their medications (numerator) by the number of clients who are experiencing pain (denominator). The client-level risk adjusters were applied as outlined by Hirdes et al. [7]. All analyses were completed using SAS software v. 9.2 (SAS Institute Inc., Cary, NC).

\section{Results}

The mean age of the sample was 83.2 years ( $\mathrm{sd}=7.6$ ), the majority were female (68.6\%) and approximately $60 \%$ of clients were divorced, separated or were widowed (Table 2). In the WRHA, a higher proportion of clients had some high school education or graduated from high school (11.7\% difference). Overall, the two provinces were very similar with respect to demographic characteristics.

The majority of home care clients (92.4\%) required full assistance with instrumental activities of daily living (IADLs). Approximately 35\% of the sample experienced ADL impairment, and nearly $50 \%$ of the clients had some degree of cognitive impairment. The two regions were very similar on the health sub-scales with the exception of the Cognitive Performance Scale in which the rate of cognitive impairment was $11.8 \%$ higher in Ontario (Table 3 ).

The top five most frequent CAPs present overall were: preventive health measures (89.6\%), IADL (81.6\%), pain (68.1\%), communication $(64.2 \%)$ and health promotion (55.7\%). The top three most frequent CAPs were identical in the two regions (Table 4). Ontario clients had higher absolute rates for $80 \%$ of the CAPs, indicating a higher level of current or future risk for these clinical issues. The opposite was seen for the brittle support CAP, which was $12.5 \%$ higher in the WRHA. This CAP identifies caregivers who are becoming stressed and at risk of not being able to continue in their caregiving role. Given that Ontario clients had higher levels of impairment generally, this finding was counterintuitive. However, we observed that clients in the WRHA were roughly $17 \%$ less likely to have a secondary informal caregiver (57.9\% vs. $74.7 \%)$ implying that their primary caregiver was likely shouldering more of the overall caregiver responsibilities and this likely explains why the CAP rate was higher in the WRHA.

\section{Home care quality indicators}

The unadjusted rates for the majority (17 of the 22) of the HCQIs were higher in Ontario than the WRHA 
Table 2 Demographic characteristics comparing Ontario and the Winnipeg Regional Health Authority $(n=111,804)$ Overall $(n=111,804) \quad$ Ontario $(n=102,554)$

$\%(n)$

\begin{tabular}{|c|c|c|c|c|}
\hline \multicolumn{5}{|l|}{ Age } \\
\hline Mean (SD) & $83.2(7.6)$ & $83.2(7.6)$ & $83.4(7.4)$ & 0.003 \\
\hline $65-74$ & $15.7(17,560)$ & $15.8(16,224)$ & $14.4(1,336)$ & 0.0007 \\
\hline $75-84$ & $40.2(44,893)$ & $40.0(41,058)$ & $41.5(3,835)$ & \\
\hline $85+$ & $44.1(49,351)$ & $44.1(45,2721)$ & $44.1(4,079)$ & \\
\hline \multicolumn{5}{|l|}{ Sex } \\
\hline Female & $68.6(76,738)$ & $68.3(70,040)$ & $72.4(6,698)$ & $<0.0001$ \\
\hline \multicolumn{5}{|l|}{ Marital status } \\
\hline Never married & $4.6(5,146)$ & $4.3(4,428)$ & $7.8(718)$ & $<0.0001$ \\
\hline Married & $34.7(38,844)$ & $35.3(36,217)$ & $28.4(2,627)$ & \\
\hline Widowed/separated/divorced & $59.9(66,974)$ & $59.6(61,139)$ & $63.1(5,835)$ & \\
\hline Other & $0.8(840)$ & $0.8(770)$ & $0.8(70)$ & \\
\hline \multicolumn{5}{|l|}{ Highest level of education } \\
\hline No schooling & $2.0(2,078)$ & $2.1(1,976)$ & $1.1(102)$ & $<0.0001$ \\
\hline Eighth grade or less & $23.8(24,316)$ & $23.8(22,028)$ & $24.7(2,288)$ & \\
\hline Some high school/high school graduate & $33.2(33,899)$ & $32.2(29,841)$ & $43.9(4,058)$ & \\
\hline Post-Secondary education & $21.5(21,943)$ & $21.6(19,993)$ & $21.1(1,950)$ & \\
\hline Unknown & $19.4(19,761)$ & $20.4(18,909)$ & $9.2(852)$ & \\
\hline
\end{tabular}

Table 3 Health and well-being comparing clients from Ontario and the WRHA $(n=111,804)$

\begin{tabular}{|c|c|c|c|c|}
\hline \multirow[t]{2}{*}{ Health sub-scales } & All $(n=111,804)$ & Ontario $(n=102,554)$ & \multirow[t]{2}{*}{ WRHA $(n=9,250)$} & \multirow[t]{2}{*}{$p$ value } \\
\hline & \multicolumn{2}{|c|}{$\%(n)$} & & \\
\hline \multicolumn{5}{|l|}{ IADL difficulty scale } \\
\hline Independent or Some Help $(0,1)$ & $7.6(8,523)$ & $7.4(7,560)$ & $10.4(963)$ & $<0.0001$ \\
\hline Full Help (2+) & $92.4(103,281)$ & $92.6(94,994)$ & $89.6(8,287)$ & \\
\hline \multicolumn{5}{|l|}{ ADL self-performance hierarchy scale } \\
\hline No impairment $(0,1)$ & $65.2(72,910)$ & $64.6(66,208)$ & $72.5(6,702)$ & $<0.0001$ \\
\hline Impairment (2+) & $34.8(38,894)$ & $35.4(36,346)$ & $27.6(2,548)$ & \\
\hline \multicolumn{5}{|l|}{ Depression rating scale } \\
\hline No signs/symptoms of depression $(0,1,2)$ & $84.3(94,270)$ & $83.8(85,898)$ & $90.5(8,372)$ & $<0.0001$ \\
\hline Signs and symptoms of depression (3+) & $15.7(17,534)$ & $16.2(16,656)$ & $9.5(878)$ & \\
\hline \multicolumn{5}{|l|}{ Cognitive performance scale } \\
\hline Intact or mild impairment $(0,1)$ & $52.2(58,304)$ & $51.2(52,479)$ & $63.0(5,825)$ & $<0.0001$ \\
\hline Cognitive impairment $(2+)$ & $47.8(53,481)$ & $48.8(50,056)$ & $37.0(3,425)$ & \\
\hline \multicolumn{5}{|l|}{ Pain scale } \\
\hline No pain/less than daily pain $(0,1)$ & $45.6(50,967)$ & $45.3(46,500)$ & $48.3(4,467)$ & $<0.0001$ \\
\hline Daily pain/severe $(2+)$ & $54.4(60,832)$ & $54.7(56,049)$ & $51.7(4,783)$ & \\
\hline \multicolumn{5}{|l|}{ CHESS scale } \\
\hline Low level of health instability $(0,1)$ & $63.0(70,371)$ & $62.3(63,883)$ & $71.1(6,488)$ & $<0.0001$ \\
\hline Mild/severe level of health instability (2+) & $37.0(41,315)$ & $37.7(38,671)$ & $29.0(2,644)$ & \\
\hline
\end{tabular}

†CHESS = Changes in Health, End-stage disease, Signs and Symptoms Scale [23]. 
Table 4 Rates for the clinical assessment protocols comparing Ontario and the WRHA $(n=111,804)$

CAPs Overall $(n=111,804) \quad$ Ontario $(n=102,554) \quad$ WRHA $(n=9,250)$

$\%(n)$

\section{Preventative health measures: immunization and screening}

Present

Not present

$89.6(91,374)$

$I A D L$

Present
Not present

Pain

Present

Not present

$68.1(76,085)$

$32.0(35,719)$

Communication disorder

Present

$64.2(71,753)$

Not present

$35.8(40,051)$

\section{Health promotion}

Present

$55.7(98,761)$

Not present

$44.3(75,351)$

Cognition

Present

$54.3(60,707)$

$45.7(51,097)$

Not present

Urinary incontinence and indwelling catheter

Present

$54.1(60,491)$

Not present

$45.9(51,313)$

Falls

Present

$49.7(55,527)$

Not present

$50.3(56,277)$

ADL/rehabilitation

Present

$46.0(51,385)$

Not present

$54.0(60,419)$

Medication management

Present

$44.0(49,220)$

$56.0(62,584)$

Not present

Psychotropic drugs

Present

$37.6(42,070)$

Not present

$62.4(69,734)$

Cardio-respiratory

Present

$35.2(39,332)$

Not present

$64.8(72,472)$

Visual function

Present

Not present

$34.7(38,837)$

$65.3(72,967)$

Skin and foot conditions

Present

$33.9(37,884)$

Not present

$66.1(73,920)$
$90.0(83,466)$

$10.0(9,284)$

$82.4(76,396)$

$17.6(16,354)$

$68.2(69,898)$

$31.8(32,656)$

$64.8(66,410)$

$35.2(36,144)$

$55.2(56,636)$

$44.8(45,918)$

$55.3(56,673)$

$44.7(45,881)$

$55.0(56,357)$

$45.1(46,197)$

$50.9(52,235)$

$49.1(50,319)$

$47.2(48,356)$

$52.9(54,198)$

$44.9(46,051)$

$55.1(56,503)$

$38.8(39,788)$

$61.2(62,766)$

$35.2(36,080)$

$64.8(66,474)$

$34.9(35,783)$

$65.1(66,771)$

$34.6(35,490)$

$65.4(67,064)$
$85.5(7,908)$

$<0.0001$

$14.5(1,342)$

$74.0(6,849)$

$<0.0001$

$26.0(2,401)$

$66.9(6,187)$

0.01

$33.1(3,063)$

$57.8(5,343)$

$<0.0001$

$42.2(3,907)$

$60.7(5,612)$

$<0.0001$

$39.3(3,638)$

$43.6(4034)$

$<0.0001$

$56.4(5,216)$

$44.7(4,134)$

$<0.0001$

$55.3(5,116)$

$35.6(3,292)$

$<0.0001$

$64.4(5,958)$

$32.8(3,029)$

$<0.0001$

$67.3(6,221)$

$34.3(3,169)$

$<0.0001$

$65.7(6,081)$

$24.7(2,282)$

$<0.0001$

$75.3(6,968)$

$35.2(3,252)$

1.0

$64.8(5,998)$

$33.0(3,054)$

0.0003

$67.0(6,196)$

$25.9(2,394)$

$<0.0001$

$74.1(6,856)$ 
Table 4 Rates for the clinical assessment protocols comparing Ontario and the WRHA $(\mathrm{n}=111,804)($ Continued)

\begin{tabular}{|c|c|c|c|c|}
\hline \multicolumn{5}{|c|}{ Pressure ulcers } \\
\hline Present & $28.3(31,649)$ & $29.2(29,934)$ & $18.5(1,715)$ & \multirow[t]{2}{*}{$<0.0001$} \\
\hline Not present & $71.7(80,155)$ & $70.8(72,620)$ & $81.5(7,535)$ & \\
\hline \multicolumn{5}{|c|}{ Depression and anxiety } \\
\hline Present & $26.2(29,274)$ & $27.0(27,645)$ & $17.6(1,629)$ & \multirow[t]{2}{*}{$<0.0001$} \\
\hline Not present & $73.8(82,530)$ & $73.0(74,909)$ & $83.0(7,621)$ & \\
\hline \multicolumn{5}{|c|}{ Institutional risk } \\
\hline Present & $25.3(28,315)$ & $26.0(26,709)$ & $17.4(1606)$ & \multirow[t]{2}{*}{$<0.0001$} \\
\hline Not present & $74.7(83,489)$ & $74.0(75,845)$ & $82.6(7,644)$ & \\
\hline \multicolumn{5}{|c|}{ Bowel management } \\
\hline Present & $23.2(25,965)$ & $24.0(24,558)$ & $15.2(1,407)$ & \multirow[t]{2}{*}{$<0.0001$} \\
\hline Not present & $76.8(85,839)$ & $76.1(77,996)$ & $84.8(7,843)$ & \\
\hline \multicolumn{5}{|l|}{ Oral health } \\
\hline Present & $19.1(21,348)$ & $19.9(20,374)$ & $10.5(974)$ & \multirow[t]{2}{*}{$<0.0001$} \\
\hline Not present & $81.9(90,456)$ & $80.1(82,180)$ & $89.5(8,276)$ & \\
\hline \multicolumn{5}{|l|}{ Nutrition } \\
\hline Present & $19.0(32,321)$ & $19.3(19,832)$ & $15.4(1,426)$ & \multirow[t]{2}{*}{$<0.0001$} \\
\hline Not present & $81.0(141,791)$ & $80.7(82,722)$ & $84.6(7,824)$ & \\
\hline \multicolumn{5}{|c|}{ Social function } \\
\hline Present & $18.1(20,193)$ & $18.0(18,460)$ & $18.7(1,733)$ & \multirow[t]{2}{*}{0.08} \\
\hline Not present & $81.9(91,611)$ & $82.0(84,094)$ & $81.3(7517)$ & \\
\hline \multicolumn{5}{|c|}{ Brittle support } \\
\hline Present & $13.1(13,367)$ & $12.0(11,103)$ & $24.5(2,264)$ & \multirow[t]{2}{*}{$<0.0001$} \\
\hline Not present & $86.9(88,633)$ & $88.0(81,647)$ & $75.5(6,986)$ & \\
\hline \multicolumn{5}{|c|}{ Reduction in formal services } \\
\hline Present & $10.8(11,026)$ & $10.7(9,904)$ & $12.1(1,122)$ & \multirow[t]{2}{*}{$<0.0001$} \\
\hline Not present & $89.2(90,974)$ & $89.3(82,846)$ & $87.9(8,128)$ & \\
\hline \multicolumn{5}{|l|}{ Behaviour } \\
\hline Present & $10.7(11,942)$ & $10.9(11,177)$ & $8.3(765)$ & \multirow[t]{2}{*}{$<0.0001$} \\
\hline Not present & $89.3(99,862)$ & $89.1(91,377)$ & $91.7(8485)$ & \\
\hline \multicolumn{5}{|c|}{ Adherence to treatment and therapies } \\
\hline Present & $6.7(7,460)$ & $6.8(6,950)$ & $5.5(510)$ & \multirow[t]{2}{*}{$<0.0001$} \\
\hline Not present & $93.3(104,344)$ & $93.2(95,604)$ & $94.5(8,740)$ & \\
\hline \multicolumn{5}{|c|}{ Environmental assessment } \\
\hline Present & $4.1(4,626)$ & $4.0(4,087)$ & $5.8(539)$ & \multirow[t]{2}{*}{$<0.0001$} \\
\hline Not present & $95.9(107,178)$ & $96.0(98,467)$ & $94.2(8,711)$ & \\
\hline Dehydration & & & & \\
\hline Present & $3.6(4,002)$ & $3.7(3,837)$ & $1.8(165)$ & $<0.0001$ \\
\hline Not present & $96.4(107,802)$ & $96.2(98,717)$ & $98.2(9,085)$ & \\
\hline Elder abuse & & & & \\
\hline Present & $2.6(2,893)$ & $2.8(2,836)$ & $0.6(57)$ & $<0.0001$ \\
\hline Not present & $97.4(108,911)$ & $97.2(99,718)$ & $99.4(9,193)$ & \\
\hline Alcohol depe & Is drinking & & & \\
\hline Present & $1.0(1,107)$ & $1.0(992)$ & $1.2(115)$ & 0.01 \\
\hline Not present & $99.0(110,697)$ & $99.0(101,562)$ & $98.8(9,135)$ & \\
\hline
\end{tabular}


Table 4 Rates for the clinical assessment protocols comparing Ontario and the WRHA $(\mathbf{n}=111,804)($ Continued)

Palliative care

$1.0(1,092)$

$1.0(1,043)$

$0.5(49)$

$<0.0001$

Not present

$99.0(110,712)$

$99.0(101,511)$

$99.5(9,201)$

(Table 5). The most prevalent HCQI in both regions was $\mathrm{ADL} /$ rehabilitation potential and not receiving therapy, followed by hospitalization in Ontario and disruptive or intense daily pain in the WRHA. The top five indicators with the largest unadjusted difference between the two provinces were: the incidence of impaired locomotion within the home (28.4\% higher in Ontario), the incidence of communication difficulty (12.7\% higher in Ontario), the prevalence of hospitalization (11.0\% higher in Ontario), the incidence of cognitive decline (10.9\% in Ontario) and the prevalence of ADL/rehabilitation potential and not receiving rehabilitation therapies, which was $24.8 \%$ higher in the WRHA.
Of the $19 \mathrm{HCQIs}$ that have client-level risk adjusters recommended, applying the risk adjustment resulted in a decrease in the difference between the adjusted rates across the majority (68.4\%) of these indicators. One exception to this rule was the triggering rate for the incidence of locomotion decline in which the adjusted difference increased by $3.2 \%$ (i.e., from $28.4 \%$ to $31.6 \%$ ). By and large, the differences between the risk adjusted rates were less than $10 \%$ between the two provinces.

\section{Discussion}

Little is publicly known about home care clients in Canada, their needs, abilities and the quality of care that

Table 5 Comparison of unadjusted and adjusted HCQI rates between the two provinces

\begin{tabular}{|c|c|c|c|c|c|c|c|}
\hline & \multicolumn{4}{|c|}{ Unadjusted rates } & \multicolumn{3}{|c|}{${ }^{*}$ Adjusted rates } \\
\hline & Ontario & $\begin{array}{c}\text { Winnipeg } \\
\%\end{array}$ & Difference & $p$ value & Ontario & $\begin{array}{c}\text { Winnipeg } \\
\%\end{array}$ & Difference \\
\hline Prevalence HCQIs & $\mathrm{n}=102,554$ & $n=9,250$ & & & $\mathrm{n}=102,554$ & $\mathrm{n}=9,250$ & \\
\hline ADL/rehabilitation potential and not receiving therapy & 66.8 & 91.6 & -24.8 & $<0.0001$ & - & - & - \\
\hline Hospitalization & 38.5 & 27.5 & 11.0 & $<0.0001$ & 27.4 & 19.6 & 7.8 \\
\hline Disruptive or intense daily pain & 35.5 & 35.0 & 0.5 & 0.29 & 35.6 & 35.7 & -0.1 \\
\hline Falls & 32.9 & 22.9 & 10.0 & $<0.0001$ & 34.1 & 25.7 & 8.4 \\
\hline No Influenza vaccination & 25.8 & 18.4 & 7.4 & $<0.0001$ & - & - & - \\
\hline Social isolation & 17.4 & 16.5 & 0.9 & 0.03 & 18.8 & 17.4 & 1.4 \\
\hline Negative mood & 16.6 & 9.9 & 6.7 & $<0.0001$ & 18.5 & 12.0 & 6.5 \\
\hline Inadequate pain control & 16.4 & 19.4 & -3.0 & 0.11 & 16.8 & 19.2 & -2.4 \\
\hline Any injuries & 9.9 & 14.0 & -4.1 & $<0.0001$ & 10.5 & 15.5 & -5.0 \\
\hline Difficulty in locomotion and no assistive device & 9.3 & 10.1 & -0.8 & $<0.0001$ & 25.2 & 22.9 & 2.3 \\
\hline Delirium & 6.6 & 5.1 & 1.5 & $<0.0001$ & 9.3 & 8.5 & 0.8 \\
\hline Weight loss & 5.9 & 3.7 & 2.2 & $<0.0001$ & 4.0 & 2.6 & 1.4 \\
\hline Inadequate meals & 3.1 & 2.3 & 0.8 & $<0.0001$ & 3.3 & 2.4 & 0.9 \\
\hline Neglect or abuse & 3.1 & 1.0 & 2.1 & $<0.0001$ & 3.8 & 1.3 & 2.5 \\
\hline Dehydration & 1.4 & 0.3 & 1.1 & $<0.0001$ & 0.8 & 0.2 & 0.6 \\
\hline No medication review by physician & 1.1 & 1.8 & -0.7 & $<0.0001$ & - & - & - \\
\hline Incidence HCQIs & $(n=14,999)$ & $(n=841)$ & & & $(n=14,999)$ & $(n=841)$ & \\
\hline Cognitive decline & 76.3 & 65.4 & 10.9 & $<0.0001$ & 76.3 & 67.9 & 8.4 \\
\hline ADL impairment & 63.7 & 57.6 & 6.1 & 0.0004 & 65.0 & 61.7 & 3.3 \\
\hline Bladder incontinence & 56.1 & 46.9 & 9.2 & $<0.0001$ & 63.3 & 56.5 & 6.8 \\
\hline Communication difficulty & 49.7 & 37.0 & 12.7 & $<0.0001$ & 46.2 & 37.5 & 8.7 \\
\hline Impaired locomotion in home & 44.3 & 15.9 & 28.4 & $<0.0001$ & 53.8 & 22.2 & 31.6 \\
\hline Skin ulcers & 8.8 & 3.6 & 5.2 & $<0.0001$ & 5.5 & 2.4 & 3.1 \\
\hline
\end{tabular}

*All risk-adjusted differences significant at $\mathrm{p}<0.0001$. 
they receive. In this sample, over $90 \%$ of clients required full assistance with IADLs, about half had some level of cognitive impairment, $54 \%$ had daily pain that could be severe at times and 35\% had ADL impairments, demonstrating the high level of need in this group. The quality indicators with the highest rates were related to clients having the potential to improve on their ADLs or having rehabilitation potential and not receiving appropriate therapies, cognitive decline, hospital use, disruptive daily pain and falls. Doran et al. [17] also used RAI-HC data to examine client safety in home care in both Ontario and in the Winnipeg area. Their measures of safety were not based on the interRAI HCQIs, but instead, focused on potential adverse events or safety issues that increase a person's risk for an adverse event. Although not identical, there is significant overlap between their adverse events or safety risks and the definitions of the HCQIs (e.g., decline in physical function, history of falls, lack of medication review among clients taking multiple medications, hospital or emergency room visits). Similar to the current findings, they also found relatively high rates of issues such as hospital visits, emergency room visits and new falls. Several other recent publications have also focused on the area of patient safety in the home care sector $[10,26,27]$. However, the adverse events, defined here as issues that are likely a result of receiving health care, often do not closely align with the HCQIs used in the current study (e.g., unexpected death, development of a urinary tract infection, diabetic foot ulcer), making comparisons difficult or inappropriate.

In the current study, we also found that clients in Ontario were more likely to experience issues such as functional decline, cognitive decline and health instability when compared to clients in the WRHA despite being very similar on basic demographic characteristics. It was expected that Ontario would have higher HCQI rates based on the higher proportion of clients experiencing impairment on the health sub-scales and the higher rates on the CAPs and this was supported in the current analysis. Following risk adjustment, Ontario continued to have higher rates across nearly all of the HCQIs, although the magnitude of the difference was typically small, and was less than $10 \%$ in most cases. Similarly, Doran et al. [17] reported slightly higher rates, without using risk adjustment, for several safety risks (e.g., decline in physical function, history of two or more falls, decline in cognition) in Ontario than in Winnipeg, and again, these differences were typically less than $10 \%$. Since the HCQIs represent potential quality issues, we cannot definitively conclude that Ontario was providing a sub-optimal level of care. Rather, the higher HCQI rates represent areas that may require further exploration to truly understand the process of care and other factors influencing these rates.
In contrast to this pattern, the $\mathrm{ADL} /$ rehabilitation potential and not receiving therapy HCQI showed a much higher rate in Winnipeg. This HCQI is made up of two parts: 1) the numerator, which includes clients who are not receiving physical therapy, occupational therapy or exercise therapy; and 2) the denominator which represents clients who trigger on the ADL CAP (i.e., clients who have the potential to benefit from rehabilitation or could improve their level of independence on ADLs). The fact that the WRHA had a higher rate on this QI could be due to their $14 \%$ lower rate on the CAP. Since the CAP makes up the denominator of the HCQI, as it decreases, the HCQI rate will go up, all other things being equal. Regardless of the driving force behind the differences observed in the current study, the sheer magnitude of the rate of this HCQI (91\% in the WRHA and $67 \%$ in Ontario) warrants further exploration. A similarly high rate was also found across 11 European countries (range: 58.2\%-98.6\%) [9]. This indicator is a flag that we need to better understand the factors influencing eligibility for rehabilitation services in the community and consider what strategies could be implemented to improve the number of clients with access to these important services.

The HCQI provincial differences decreased for the majority of the HCQI rates following risk adjustment. In the instances where risk adjustment was applied and the provincial differences increased, the differences did not exceed $4 \%$. This is very similar to earlier work by Dalby et al. [8]. Client-level risk adjustment appears to reduce the "extremes" in the rates, bringing both high and low rates closer to the centre. The fact that Ontario continued to have higher adjusted rates could indicate that other types of risk adjustment are warranted (e.g., agency-level risk adjustment) or it could reflect variations in processes of care that resulted in different outcomes between the two regions. However, the fact that most of the adjusted rates showed differences that were very small implies that there were likely no substantial variations in quality of care between these two regions. Determining how processes of care or additional risk adjustment might have influenced the rates was beyond the scope of the current study.

One interesting departure from previous research was our finding that the HCQI related to the incidence of impaired locomotion in the home had an adjusted difference that was $32 \%$ higher in Ontario. This is approximately $20 \%$ higher than what has been previously reported using RAI-HC data and the same methodology [8]. This may be a reflection of access to assistive devices to support locomotion in the home. Another similar HCQI, namely, the prevalence of difficulty in locomotion but not having an assistive device showed virtually no difference between the two regions, both 
before and following risk adjustment. So, it does not appear that the higher rate for the incidence HCQI can easily be explained by a lack of service provision for Ontario clients. It is possible that the risk adjusters for the incidence HCQI (e.g., difficulties with dressing, reduction in physical activity and cognitive impairment) are not adequately taking into account other factors that could increase this rate regardless of the quality of care. Further analysis may be warranted, at least for this particular indicator, to determine if other risk adjusters are relevant.

A limitation of this study was the inability to align the RAI-HC assessment data between the two regions. Only one year of complete data for the WRHA (20062007) was available, and data for this time frame were not available for Ontario. As such, we chose to use the most recent $\mathrm{RAI}-\mathrm{HC}$ data in the two provinces. It is recognized that for an indicator like the prevalence of not receiving an influenza vaccination annual variations could be important. However, for the remaining indicators, it is unlikely that the rates would change dramatically from year to year. Given that the differences between the adjusted rates comparing Ontario and Winnipeg were small (typically differences of less than $10 \%$ ), this provides some evidence that the timeframe discrepancy did not substantially influence the results.

This study was also limited by using only client-level risk adjustment. Agency-level risk adjustment has also been shown to have an influence on minimizing the HCQI rates between different regions [8]. Agency-level information such as characteristics of clients newly admitted into the home care program was not available through the existing database so an agency-level risk adjustment could not be completed. Caution must therefore be used when interpreting the results from this study because not all types of risk adjustment have been conducted. As such, there may still be other characteristics of the organization that could influence the HCQI rates. The entire area of risk adjustment for health care quality indicators is very new and little evidence exists to support one particular method over another $[8,9]$.

Finally, the RAI-HC data are only available for longstay clients, thereby introducing a possible selection bias. These clients may be more likely to experience functional and cognitive impairment, which could influence the HCQI rates. It is not appropriate to evaluate the quality of care for short-stay clients using the HCQIs as this could potentially penalize organizations who have had a very limited amount of time within which to assess the client and implement a care plan. As such, focusing on long-stay clients, when generating the HCQIs, is the most reasonable approach.

\section{Conclusions}

This research utilized a near census of clients from Ontario and a very large sample of clients from the WRHA in order to bridge the gap in the home care literature with respect to understanding the needs of home care clients in Canada and the key quality issues identified with a set of HCQIs. Home care organizations can utilize the HCQIs to identify potential quality issues within their own region and also to compare their results to other parts of Canada utilizing the RAI-HC. As more regions begin to submit RAI-HC data to the Canadian Institute for Health Information, the potential will increase substantially for the HCQI rates to be generated for a number of provinces and territories and for these regions to begin to utilize their data in ways that support continuous quality improvement. The HCQIs represent a practical decision-support tool, collected at the point of care, that provide a means for home care providers and policy makers to assess and compare quality as part of the ongoing commitment to continually enhance the care provided in the community.

\section{Abbreviations}

ADLs: Activities of Daily Living; HCQIs: Home Care Quality Indicators; IADLs: Instrumental ADLs; QI: Quality Indicator; RAI-HC: Resident Assessment Instrument for Home Care; WRHA: Winnipeg Regional Health Authority.

\section{Competing interests}

The authors declare that they have no competing interests.

\section{Authors' contributions}

AM led the data analysis and took the lead on manuscript development. DG was involved in data analysis and preparation and review of the manuscript. Both authors read and approved the final manuscript.

\section{Acknowledgements}

The authors would like to acknowledge the Canadian Institute for Health Information for access to these data through the Graduate Student Data Access Program.

Received: 14 May 2013 Accepted: 22 January 2014

Published: 25 January 2014

\section{References}

1. Canadian Home Care Association: Portraits of home care in Canada. Mississauga, Ontario: Canadian Home Care Association; 2008:1-20.

2. Canadian Health Care Association: From the Margins to the Mainstream: Portraits of home care in Canada 2008. Ottawa, Ontario: Canadian Health Care Association; 2008

3. Canadian Institute for Health Information: Health Care in Canada, 2011: A focus on seniors and Aging. Ottawa, Ontario; 2011.

4. The National Association for Home Care \& Hospice: Basic statistics about home care. Washington, DC: National Association for Home Care \& Hospice; 2010:1-14.

5. Bernstein AB, Hing E, Moss AJ, Allen KF, Siller AB, Tiggle RB: Health care in America: Trends in utilization. Hyattsville, Maryland: National Center for Health Statistics; 2003.

6. Masotti P, Green M, Shortt S, Hunter D, Szala-Meneok K: (Eds):Adverse events in community care: developing a research agenda. Healthc $Q$ 2007, 10:63-69.

7. Hirdes JP, Fries BE, Morris JN, Ikegami N, Zimmerman D, Dalby DM, et al: Home care quality indicators (HCQIs) based on the MDS-HC. Gerontologist 2004, 44:665-679. 
8. Dalby DM, Hirdes JP, Fries BE: Risk adjustment methods for home care quality indicators (HCQIs) based on the minimum data set for home care. BMC Health Serv Res 2005, 5:7.

9. Bos JT, Frijters DHM, Wagner C, Carpenter Gl, Finne-Soveri H, Topinkova E, et al: Variations in quality of home care between sites across Europe, as measured by home care quality indicators. Aging Clin Exp Res 2007. 19:323-329.

10. Madigan EA: A description of adverse events in home healthcare. Home Healthc Nurse 2007, 25:191-197.

11. Health Canada: Canada health act - annual report 2008-2009. Ottawa, Ontario: Health Canada; 2009:1-240.

12. Morris J: interRAI HC - home care. interRAl 2007, 2011:3-16.

13. Morris JN, Bernabei R, Ikegami N, Gilgen R, Frijters D, Hirdes JP, et al: RAl-Home Care (RAl-HC) Assessment Manual for Version 2.0. Washington, DC: interRAI Corporation; 1999.

14. lezzoni LI: Risk adjustment for measuring health care outcomes. Chicago, Illinois: Health Administration Press; 2003.

15. Dalby DM, Hirdes JP: The relationship between agency characteristics and quality of home care. Home Health Care Serv Q 2008, 27:59-74.

16. Health Quality Ontario [internet. Toronto, Ontario; 2011. [cited 2014 Jan. 23]. Available from http://www.hqontario.ca/public-reporting/home-care.

17. Doran DM, Hirdes J, Blais R, Baker GR, Pickard J, Jantzi M: The nature of safety problems among Canadian homecare clients: evidence from the RAI-HC reporting system. J Nurs Manag 2009, 17:165-174.

18. Burrows AB, Morris JN, Simon SE, Hirdes JP, Phillips CD: Development of an MDS-based depression rating scale for use in nursing homes. Age Ageing 2000, 29:165-172.

19. Martin L, Poss JW, Hirdes JP, Jones RN, Stones MJ, Fries BE: Predictors of a new depression diagnosis among older adults admitted to complex continuing care: implications for the Depression Rating Scale (DRS). Age Ageing 2008, 37:51-56.

20. Morris JN, Fries BE, Mehr DR, Hawes C, Mor V, Lipsitz L: MDS cognitive performance scale. J Gerontol A Biol Sci Med Sci 1994, 49:M174-M182.

21. Hartmaier SL, Sloane PD, Guess HA, Koch GG, Mitchell CM, Phillips CD: Validation of the minimum data set cognitive performance scale: agreement with the mini-mental state examination. I Gerontol A Biol Sci Med Sci 1995, 50A:M128-M133.

22. Morris JN, Fries BE, Morris SA: Scaling ADLs within the MDS. J Gerontol A Biol Sci Med Sci 1999, 54A:M546-M553.

23. Hirdes JP, Frijters DH, Teare GF: The MDS-CHESS scale: a new measure to predict mortality in institutionalized older people. J Am Geriatr Soc 2003, 51:96-100.

24. Armstrong JJ, Stolee P, Hirdes JP, Poss JW: Examining three frailty conceptualizations in their ability to predict negative outcomes for home-care clients. Age Ageing 2010, 39:755-758.

25. Fries BE, Simon SE, Morris JN, Flodstrom C, Bookstein FL: Pain in US nursing homes: validating a pain scale for the minimum data set. Gerontologist 2001, 41:173-179.

26. Doran DM, Hirdes JP, Blais R, Baker GR, Poss JW, Li X, et al: Adverse events among Ontario home care clients associated with emergency room visit or hospitalization: a retrospective cohort study. BMC Health Serv Res 2013, 13:227.

27. Blais R, Sears NA, Doran DM, Baker GR, Macdonald M, Mitchell L, et al: Assessing adverse events among home care clients in three Canadian provinces using chart review. BMJ Quality and Safety 2013, 22:989-997.

doi:10.1186/1472-6963-14-37

Cite this article as: Mofina and Guthrie: A comparison of home care quality indicator rates in two Canadian provinces. BMC Health Services Research 2014 14:37.

\section{Submit your next manuscript to BioMed Central and take full advantage of:}

- Convenient online submission

- Thorough peer review

- No space constraints or color figure charges

- Immediate publication on acceptance

- Inclusion in PubMed, CAS, Scopus and Google Scholar

- Research which is freely available for redistribution

Submit your manuscript at www.biomedcentral.com/submit
Ciomed Central 